\title{
Effects of different fatty acid profiles on markers of endothelial function in cultured human dermal microvascular endothelial cells
}

\author{
S. C. Cottin, R. C. Siow, T. A. Sanders and W. L. Hall \\ Nutritional Sciences Division, School of Biomedical and Health Sciences, King's College London, 150 Stamford Street, \\ London SE1 9NH, UK
}

Elevated NEFA plasma levels are associated with insulin-resistant states and are involved in endothelial dysfunction ${ }^{(1)}$. Some evidence suggests that the composition of circulating NEFA may influence endothelial function ${ }^{(2)}$. Here, we assess the effect of four different fatty acid (FA) profiles, representing blood levels typically reported following dietary patterns, on markers of endothelial function in vitro, prostacyclin $\left(\mathrm{PGI}_{2}\right)$ and nitric oxide (NO) production. SFA (16:0 and 18:0), MUFA (18:1n-9) and PUFA (18:2n-6, 20:4n-6, $20: 5 n-3$ and $22: 6 n-3$ ) were complexed with bovine serum albumin (BSA) to obtain a molar ratio of $2.5: 1$ and combined in different proportions to obtain four different FA profiles so that they are either rich in SFA, MUFA, $n-6$ PUFA or $n-3$ PUFA. Human dermal microvascular endothelial cells (HDMEC) were incubated with each FA profile for $24 \mathrm{~h}$ at a normal $(400 \mu \mathrm{M})$ or higher concentration $(1000 \mu \mathrm{M})$, corresponding to healthy and insulin-resistant plasma levels, respectively, with or without $\mathrm{TNF} \alpha(24 \mathrm{~h}, 10 \mathrm{ng} / \mathrm{ml}) \mathrm{or}$ insulin $(2 \mathrm{~h}, 1 \mu \mathrm{g} / \mathrm{ml})$, in four replicate experiments. Endothelial function was assessed by measuring concentrations in the medium of NO by fluorometric assay (Cayman Europe) and $\mathrm{PGI}_{2}$ by ELISA (Cayman Europe), and concentrations were adjusted to the total cell protein content measured by BCA assay (Pierce). There was no difference in NO production following different FA profiles, neither at higher or normal concentrations. The high $n-6$ PUFA profile significantly increased $\mathrm{PGI}_{2}$ production $(P<0.01)$, compared to MUFA and SFA profiles. The high $n-3$ PUFA profile decreased $\mathrm{PGI}_{2}$ production compared to other FA profiles $(P=0.053)$ (Figure). There was no difference between the higher and normal FA concentrations in their effects on $\mathrm{PGI}_{2}$ production.

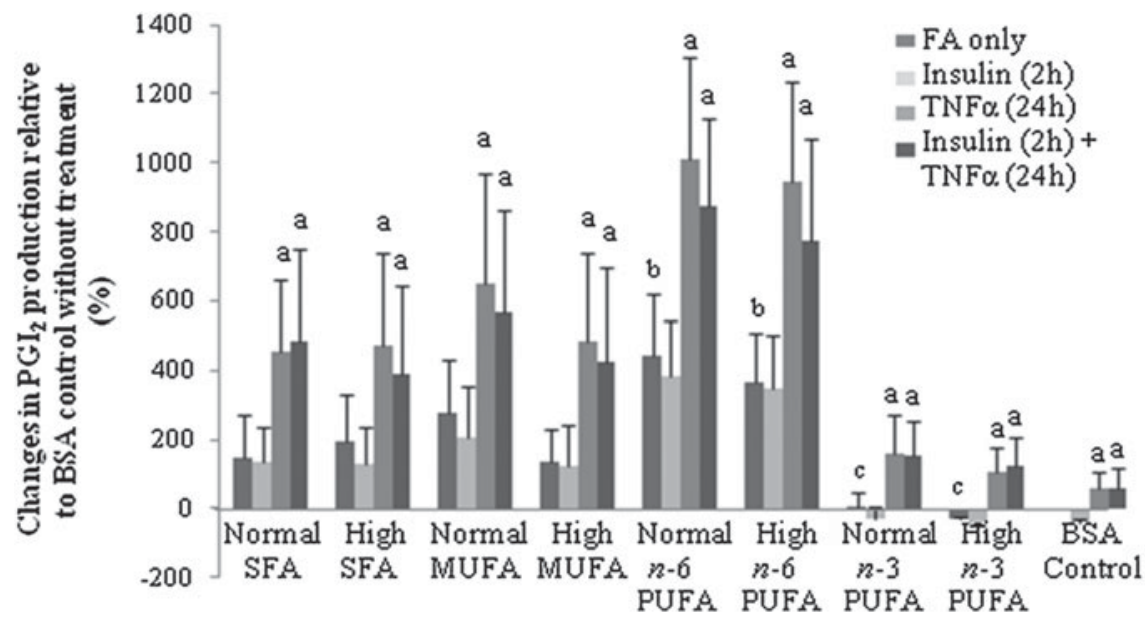

Percentage change in $\mathrm{PGI}_{2}$ production by HDMEC following $24 \mathrm{~h}$ incubation with 400 or $1000 \mu \mathrm{M}$ fatty acid mixtures rich in either SPA, MUFA, $n$ - 6 PUFA or $n-3$ PUFA, in the presence or absence of TNF- $\alpha$ and insulin. Values are means with their SD represented by vertical bars. a Indicated significant increase in PGI 2 production compared to FA only in each profile $(P<0.01)$. b Indicated significant increase in $\mathrm{PGI}_{2}$ production in the $n-6$ PUFA profile compared to all the other profiles in the absence of TNF $\alpha$ or insulin $(P=<0.01)$. c Indicated decrease in PGI $_{2}$ production in the $n-3$ PUFA profile compared to the SFA, MUFA, $n-6$ PUFA profile in the absence of TNF $\alpha$ or insulin $(P=0.054)$

In all profiles, $\mathrm{PGI}_{2}$ was significantly increased by $\mathrm{TNF} \alpha(P<0.01)$, but unchanged by insulin treatment; there was no interaction between the FA profile and inflammatory stimulus. The data from the study are the first in vitro study to show that treatment of endothelial cells with physiological concentrations of FA added as profiles representing those found circulating in the blood may influence the vasodilatory function of the microvascular endothelium. This will further our understanding of the effects of circulating NEFA on endothelial function.

1. Steinberg HO \& Baron AD (2002) Vascular function, insulin resistance and fatty acids. Diabetologia 45, 623-634.

2. Yli-Jama P, Seljeflot I, Meyer HE et al. (2002) Serum non-esterified very long-chain PUFA are associated with markers of endothelial dysfunction. Atherosclerosis 164, 275-281. 